\title{
Simulation Analysis of Absorptive Chaff Cloud Jamming Performance on Radar Wave
}

\author{
Jinheng Yang ${ }^{\mathrm{a})}$, Xing Wang ${ }^{\mathrm{b})}$, Xiaotian $\mathrm{Wu}^{\mathrm{c})}$ and Xiaoxuan Dong ${ }^{\mathrm{c})}$ \\ ${ }^{1}$ Air Force Engineering University, Xi'an 710038, China. \\ a)kellyyoung331@163.com \\ b)13399289501@189.cn \\ c)celeswu1212@sina.com
}

\begin{abstract}
Absorptive chaff is a light and high-efficiency kind of passive jamming material, which can decline echo amplitude enemy detection that equipment receives largely scattered in the air, thus reducing the probability of being found and tracked by radar equipment. In this paper, the jamming performance of absorptive chaff cloud on radar wave is studied. First, combining the existing research result, the jamming mechanism of absorptive chaff cloud on radar wave is analyzed. Then, according to transmission-line theory and energy conservation principles, the models of reflection loss and transmission loss on the surface of absorber. Finally, the power attenuation model is built, according to which, how radar maximum range changes after interference is obtained. Simulation results show that, absorptive chaff cloud has obvious weaken effect on radar wave. With 100 meters' thickness, the attenuation can reach $23.4 \mathrm{~dB}$, which has some value of application in the battleground.
\end{abstract}

Keywords: Absorptive, Chaff Cloud, Jamming, Radar Wave, Reflection Loss, Transmission Loss

\section{INTRODUCTION}

In information war, radar electronic countermeasure (ECM) is an essential means of self-defense that weapon system and military target need for their survival and development. Passive jamming is an electronic countermeasure with the advantages of simple equipment manufacturing, easy to use and low price, which doesn't radiate energy by itself, but scatters or absorbs electromagnetic wave through the medium in order to disrupt enemy's radar. Releasing chaff is a significant part of passive jamming against radar guidance weapon system [1].

Traditional chaff jamming works through scattering principal, which creates secondary radiation on the chaff in the role of alternating current, thus causing passive jamming. With the development of modern electronic information technology, the accuracy and performance of new radar system and advanced guided weapon are greatly improved. Facing these threats, traditional chaff jamming alone might expose some limitations in the battleground:

1. The shortage of priori knowledge might disable chaff jamming.

2. The false target that chaff cloud generates might be recognized by new radar system such as MTI or variable polarization radar.

3. In centroid jamming, in order to achieve ideal results, the scattering echo of chaff cloud and target need to be in the same impulse unit.

In conclusion, even if traditional chaff jamming has shown good tactical performance in modern electronic warfare, its limitations might become our Achilles' heel. Therefore, in the face of constantly developing radar technology, it has great significance to develop a new, practical and low-cost absorptive chaff to deal with enemy's radar wave. 
At present, the related field has started the research on absorptive chaff. Reference [2] analyzes the jamming mechanism of absorptive chaff and studies the preparation of new absorptive chaff by wet chemical method, but it hasn't made jamming effectiveness analysis aiming at specific scene or target. Reference [3] only creates the scattering, absorptive and attenuation cross section expression of single fiber with finite conductivity, which has poor universality with too many restricted conditions. In reference [4], the radar reflection characteristics of multi-orientation iron-fiber absorbing materials are analyzed, in which the application in absorptive chaff hasn't been taken into consideration. Reference [5] puts forward the model of power attenuation of radar signal on the background of aeroplane laying jamming corridor only with absorptive chaff. Aiming at disadvantages of references above, this paper firstly presents the process that absorptive chaff cloud disrupts radar wave after analyzing its jamming mechanism. Then it simulates the jamming effects of absorptive chaff cloud. Finally, the practical application is preliminarily discussed.

\section{Derivation of Mechanism of Absorptive Chaff}

Due to the jamming mechanism - absorption attenuation instead of scattering, it has lower demand for priori knowledge than the traditional one. In actual application, different tactics can be taken based on different combat target or mission. Not only can absorptive chaff be used alone to lay absorptive jamming corridor, but it can form a complementary structure with traditional one.

As is known, electromagnetic wave propagation in absorber is complex, including absorption, diffraction, reflection and so on. Aiming at the problem, this paper learned from the method that Gauss dealt with flux, which is to see the whole absorber as the research object, or study micro issue from a macro viewpoint. According to energy conservation principles, energy flowing in and out can be analyzed through outside surface of the absorber.

From the micro perspective, absorptive chaffs are not distributed evenly in the air. But macroscopically speaking, the distribution can be regarded as isotropic materials. As shown in Fig. 1, when incident electromagnetic wave, whose field intensity is $\boldsymbol{E}^{+}$, enters absorber, the wave along direction $\mathbf{z}$ (or $\mathbf{- z}$ ) will be generated at the border of $z=0$ (or $z=-d$ ), which is called transmitted wave $E_{t 0}$ (or reflected wave $E_{r 0}$ ) with field intensity $T_{C} \boldsymbol{E}^{+}$(or $R_{C} \boldsymbol{E}^{+}$). $T_{C}$ is called transmission loss and $R_{C}$ is called reflection loss. Apostrophes represent the complex transmission of electromagnetic wave in the absorber.

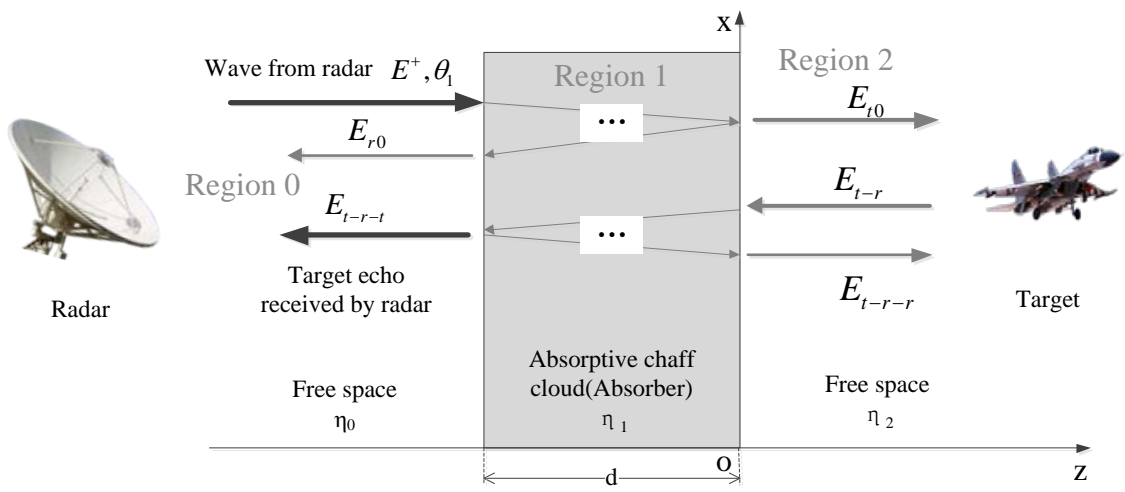

FIGURE 1. Schematic diagram of absorptive chaff cloud interfering radar wave.

Next, take transmission loss as an example, whose derivation process is shown blew.

Based on transmission-line theory of electromagnetic wave, we can get:

$$
\begin{aligned}
E_{t 0}= & E^{+} T_{01} T_{12} \mathrm{e}^{-\gamma_{1} d} \\
& +E^{+} T_{01} T_{12} \mathrm{e}^{-\gamma_{1} d} R_{12} R_{10} \mathrm{e}^{-2 \gamma_{1} d} \\
& +E^{+} T_{01} T_{12} \mathrm{e}^{-\gamma_{1} d}\left(R_{12} R_{10} \mathrm{e}^{-2 \gamma_{1} d}\right)^{2} \\
& \cdots \\
& +E^{+} T_{01} T_{12} \mathrm{e}^{-\gamma_{1} d}\left(R_{12} R_{10} \mathrm{e}^{-2 \gamma_{1} d}\right)^{n-1}
\end{aligned}
$$

In the expression above, $\mathrm{d}$ is the thickness of absorber, $\gamma_{1}$ is complex propagation constant of absorber:

$$
\gamma_{1}=j \omega \sqrt{\mu_{C} \varepsilon_{C}}
$$


The real part of $\gamma_{1}$ is called attenuation constant while the imaginary part is called phase constant. $\varepsilon_{C}$ and $\mu_{C}$ are effective electromagnetic parameters, which influence the absorption properties of the absorber directly. $R_{i j}$ and $T_{i j}(i, j=0,1,2)$ are reflection coefficient and transmission coefficient, indicating when electromagnetic wave enters the border between region $i$ and region $j$ from region $i$, reflected wave is generated in region $i$ while transmitted wave is generated in region $j$, as shown in:

$$
R_{i j}=\frac{\eta_{j}-\eta_{i}}{\eta_{i}+\eta_{j}}, \quad T_{i j}=\frac{2 \eta_{j}}{\eta_{i}+\eta_{j}}
$$

In the expression (3), $\quad \eta_{i}$ and $\eta_{j}$ are characteristic impedance of the absorber $(i, j=1,2)$.

According to Effective Medium Theory, for a two-component composite material, its effective electromagnetic parameters can be figured out through electromagnetic parameter of each component and corresponding volume fraction. The absorptive chaff cloud can be regarded as the absorber using air as substrate and chaffs as filling materials, in which volume fraction of filling materials is:

$$
\theta_{1}=n V_{s}(4)
$$

$n$ is number of chaffs per unit volume, or concentration. $V_{S}$ is single chaff volume.

Based on Looyenga equation, effective electromagnetic parameters of absorptive chaff are given as:

$$
\left\{\begin{array}{l}
\mu_{C}{ }^{1 / 3}=\theta_{1} \mu_{1}^{1 / 3}+\left(1-\theta_{1}\right) \mu_{0}^{1 / 3} \\
\varepsilon_{C}{ }^{1 / 3}=\theta_{1} \varepsilon_{1}^{1 / 3}+\left(1-\theta_{1}\right) \varepsilon_{0}^{1 / 3}
\end{array}\right.
$$

Subscript ' 1 ' represents absorptive material and ' 0 ' represents free space.

From expression (1), the total field intensity of transmitted wave can be expressed as:

$$
\begin{aligned}
E_{t 0} & =T_{C} E^{+} \\
& =T_{01} T_{12} \mathrm{e}^{-\gamma_{1} d}\left(1+\lim _{n \rightarrow \infty} \sum_{i=1}^{n-1}\left(R_{12} R_{10} \mathrm{e}^{-2 \gamma_{1} d}\right)^{i}\right) E^{+} \\
& =T_{01} T_{12} \mathrm{e}^{-\gamma_{1} d}\left(\sum_{i=0}^{\infty}\left(R_{12} R_{10} \mathrm{e}^{-2 \gamma_{1} d}\right)^{i}\right) E^{+}
\end{aligned}
$$

Considering that $R_{12} R_{10}<1$, transmission loss is expressed as:

$$
T_{C}=\frac{T_{01} T_{12} \mathrm{e}^{-\gamma_{1} d}}{1-R_{12} R_{10} \mathrm{e}^{-2 \gamma_{1} d}}
$$

Similarly, reflection loss can be solved from:

$$
R_{C}=\frac{E_{r 0}}{E^{+}}=R_{01}+\frac{T_{01} T_{10} R_{12} e^{-2 \gamma_{1} d}}{1-R_{12} R_{10} e^{-2 \gamma_{1} d}}
$$

In general, values of transmission and reflection loss are presented in decibels, record:

$$
\left\{\begin{array}{l}
T_{C}{ }^{\prime}=20 \lg \left|T_{C}\right| \\
R_{C}{ }^{\prime}=20 \lg \left|R_{C}\right|
\end{array}\right.
$$

\section{Analysis of Jamming Radar Wave}

According to radar principles, under the condition of ideal free space, the echo power that monostatic radar receives can be given as:

$$
P_{r}=\frac{P_{t} G_{t} \sigma A_{e}}{\left(4 \pi R^{2}\right)^{2}}
$$

$P_{t}$ is radar transmission power, $G_{t}$ is radar antenna gain, $A_{e}$ is effective aperture of radar antenna, $\sigma$ is radar cross section or RCS of the target and $R$ is distance from radar antenna to target. Particularly, when the same antenna plays the role of both transmission and reception, antenna gain $G_{t}$ and transmission power $P_{t}$ have the relationship below:

$$
A_{e}=\frac{G_{t} \lambda^{2}}{4 \pi}
$$

Fig. 1 shows the procedures from radar transmitting electromagnetic wave to receiving echo wave from the target, when absorptive chaff clouds is in the working. The specific procedures are shown blew: 
1) Radar wave, whose field intensity is $\boldsymbol{E}^{+}$, enters the jamming cloud before chaff cloud's reflected wave $E_{r 0}$ and transmitted wave $E_{t 0}$ are generated at $z=-d$ and $z=0$ :

$$
\left\{\begin{array}{l}
E_{r 0}=E^{+} \cdot R_{C} \\
E_{t 0}=E^{+} \cdot T_{C}
\end{array}\right.
$$

2) A portion of transmitted wave $E_{t 0}$ turns into scattering wave at the surface of the target, some of which $\left(E_{t-r}\right)$ enters chaff cloud again, forming radar receiving wave $E_{t-r-t}$ and chaff cloud echo $E_{t-r-r}$ :

$$
\left\{\begin{array}{l}
E_{t-r-r}=E_{t-r} \cdot R_{C} \\
E_{t-r-t}=E_{t-r} \cdot T_{C}
\end{array}\right.
$$

Power value of the scattering wave from the target is shown as:

$$
P_{t-r}=\frac{P_{t} G_{t} T_{C}^{2} \sigma}{4 \pi R^{2}}
$$

The scattering wave entering and going through chaff cloud again is received by radar, whose power value is as follows:

$$
P_{t-r-t 0}=P_{t-r} T_{C}^{2} \frac{A_{e}}{4 \pi R^{2}}
$$

In general, jamming corridor covers a wide area and the distance between target and chaff cloud $R_{1}<<R$, so it is believed that all target scattering echo goes into chaff cloud. The echo formed the first time by target scattering wave radiates target again and scatters, whose received power value is:

$$
P_{t-r-t 1}=P_{t-r} R_{C}^{2} \cdot \frac{\sigma}{4 \pi R_{1}^{2}} \cdot T_{C}^{2} \frac{A_{e}}{4 \pi R^{2}}
$$

3) Chaff cloud echo $E_{t-r-r}$ will go on with step 2). Each time the echo forming in region 2 will start the next cycle, some of which turn into the transmitted wave in region 0 , that target echoes are added together and then received by radar antenna:

$$
P_{t-r-t \mathrm{~N}}=P_{t-r} T_{C}^{2} \frac{A_{e}}{4 \pi R^{2}}\left(\frac{R_{C}^{2} \sigma}{4 \pi R_{1}^{2}}\right)^{\mathrm{N}}, \quad N=0,1,2, \ldots
$$

$d_{1}$ is distance between target and jamming cloud. $N$ represents $\mathrm{N}$ time target scattering echo forms the reflection at $z=0$.

4) Finally, electromagnetic wave received by radar consists of reflected wave $E_{r 0}$ that forms when firstly entering cloud and target echoes $E_{\Sigma r}$ that were added together, whose power expression is $P_{r}{ }^{\prime}=P_{r 0}+P_{\Sigma r}$. According to expression (12) (17), there is:

$$
P_{r}^{\prime}=\frac{P_{t} G_{t}^{2} R_{C}^{2} \lambda^{2}}{\left(4 \pi R^{2}\right)^{2}}+\frac{P_{t} G_{t}^{2} T_{C}^{4} \lambda^{2} \sigma}{\left(1-\frac{R_{C}^{2} \sigma}{4 \pi R_{1}^{2}}\right)(4 \pi)^{3} R^{4}}
$$

$\sigma_{0}$ is effective RCS of chaff cloud. Set $P_{r}$ ' as sensitivity of radar receiver $\left(S_{\min }\right)$, then $R=R_{\text {max }}^{\prime}$, which is radar's maximum range under the jamming of absorptive chaff as shown in:

$$
R_{\text {max }}^{\prime}=\sqrt[4]{\frac{P_{t} G_{t}^{2} \lambda^{2}}{S_{\min }(4 \pi)^{3}}\left(4 \pi R_{C}^{2}+\frac{T_{C}^{4} \sigma}{1-\frac{R_{C}^{2} \sigma}{4 \pi R_{1}^{2}}}\right)}
$$

According to expression (10) and (18):

$$
\frac{P_{r}}{P_{r}{ }^{\prime}}=\frac{\sigma}{4 \pi R_{C}^{2}+\frac{T_{C}^{4} \sigma}{1-\frac{R_{C}^{2} \sigma}{4 \pi R_{1}^{2}}}}
$$

To express how absorptive chaff cloud affects radar's maximum range, define attenuation loss as $L$, there is:

$$
L=10 \lg \frac{P_{r}}{P_{r}{ }^{\prime}}
$$

Since $L=2 \beta R$, then:

$$
P_{r}{ }^{\prime}=P_{r} e^{-0.115 \beta R}
$$


As the received power value reduces due to absorptive chaff cloud, radar's maximum range is shortened to:

$$
R_{\text {max }}^{\prime}=\sqrt[4]{\frac{P_{t} G_{t}^{2} \lambda^{2} \sigma}{(4 \pi)^{3} S_{\text {min }}}} e^{-0.029 \beta R_{\text {max }}^{\prime}}
$$

In the last expression, $e^{-0.029 \beta R_{\text {max }}^{\prime}}$ is called attenuation factor.

\section{Simulation Results and Analysis}

Ignore atmospheric attenuation, according to expression (1) (9), transmission loss and reflection loss of absorptive chaff cloud are shown in Fig. 2. From Fig. 2, with the increase in thickness, transmission loss descends linearity while reflection loss is in periodic oscillation between -91dB -73dB. The oscillation is caused by interference of electromagnetic wave reflected on two surface of absorber along the incident direction [6], which is related to concentration of filling materials and frequency of incident wave. It will weaken until it's gone with the increase in thickness. And reflection loss tends to be stable (about -78dB). Obviously, value of reflection loss is small and approximately three orders of magnitude smaller than transmission loss.

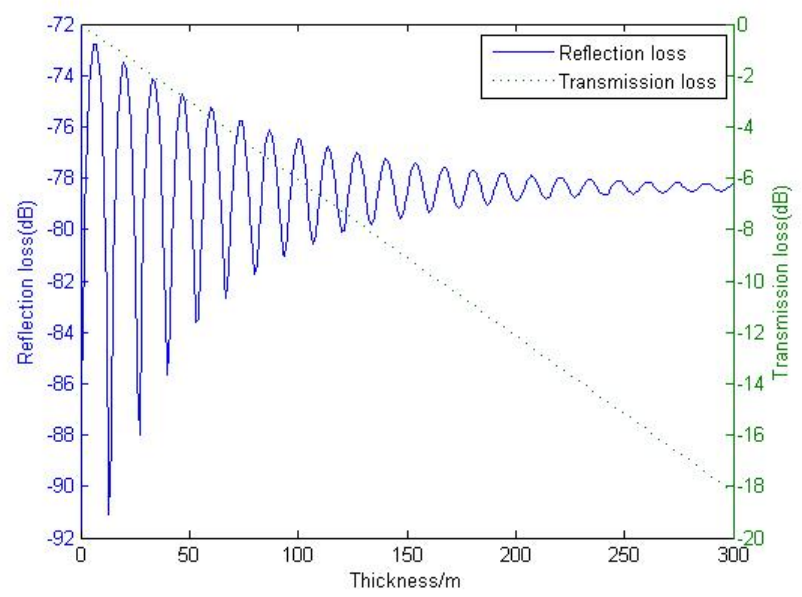

FIGURE 2. Reflection loss and transmission loss.

Fig. 3 shows the impact of different average attenuation coefficient on radar's maximum range. The horizontal axis represents radar's maximum range when chaff jamming exists, and the vertical axis represents radar's maximum range when chaff jamming doesn't exist. The curve takes average attenuation coefficient as parameter and different $\beta$ correspond to one different exponential curve. With the increase in average attenuation coefficient, the slope increases as well. If $\beta$ is known, radar's maximum range under chaff jamming can be estimated from Fig. 3.

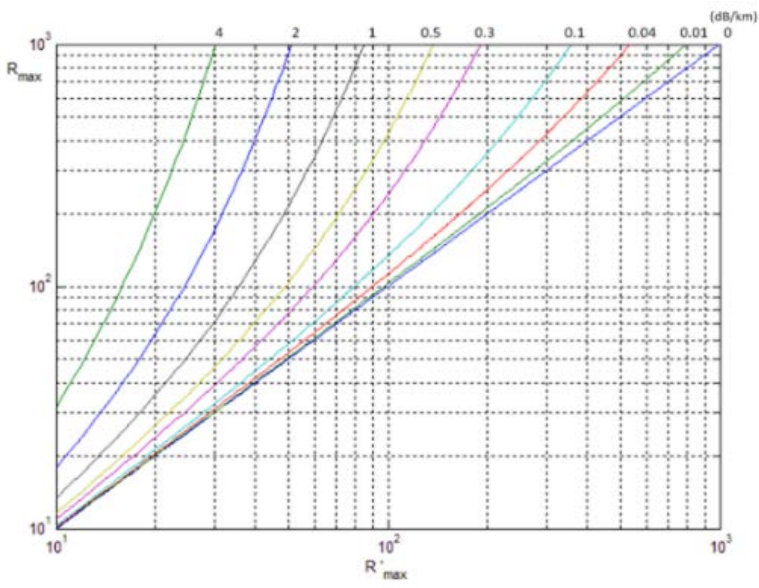

FIGURE 3. Radar range curve after jamming.

On the other hand, when there is some priori knowledge on enemy's radar, some proper preparations can be 
made according to operational environment and requirement. For instance, crews may change proportioning of materials, mix it with traditional chaffs, preset the launch mode according to task characteristics and conduct Active-Passive-Complex ECM, aiming to weaken enemy radar's energy or decrease radar's maximum range thus protecting fighters from damage.

In aviation, the frequency of airborne radar equipment is usually distributed over the range between $8 \sim 18 \mathrm{GHz}$. So take a typical value $-10 \mathrm{GHz}$ as simulation condition, the result is shown as Table 1 :

TABLE 1. Absorbing attenuation under electromagnetic wave.

\begin{tabular}{c|cccccc}
\hline $\begin{array}{c}\text { Volume } \\
\text { fraction }\end{array}$ & 50 & 100 & 150 & 200 & 250 & 300 \\
\hline $1.2 \times 10^{-4}$ & 5 & 10 & 15 & 20 & 15 & 30 \\
$1.6 \times 10^{-4}$ & 6.7 & 13.3 & 20 & 26.7 & 33.3 & 40 \\
$2.0 \times 10^{-4}$ & 8.3 & 16.7 & 25 & 33.3 & 41.7 & 50 \\
\hline
\end{tabular}

In Table 1, when thickness is $150 \mathrm{~m}$, power attenuation loss of the jamming cloud with materials' volume fraction of $1.6 * 10^{-4}$ can reach $20 \mathrm{~dB}$. As the thickness is increasing, the corresponding absorbing property is improved.

In battlefield applications, to insure that chaffs have enough concentration after spread, some measures might be taken, such as continuously releasing and multi-fighter jointly releasing, which avoids a lot of waste of resources under the premise of jamming effect. On the other hand, after the long diffusion, concentration will decrease, while keeping the number of chaffs. So the number of chaffs released once is important to whether jamming works or not. In the process of development and production, choosing appropriate granularity of absorbing material or the number of chaffs in the single jamming cartridge can provide strong support for multitasking. For the former, the smaller granularity is, the higher specific surface area it will get. Specially, when it decreases to nanoscale, absorbing material's quantum size effect and high surface atomic ratio can create new absorption channel, thus improving absorbing property.

\section{CONCLUSION}

By regarding absorptive chaff cloud as single layer absorber, this paper analyzes the jamming mechanism of the cloud and builts the power attenuation model based on transmission-line theory and energy conservation principles. How radar maximum range changes after jamming is obtained under different thickness, frequency and volume fraction. Combining practical application, there are some reference values to the development and production, as well as combat operation.

In the future, as for the research direction of absorptive chaff cloud, these points blew can be considered:

1) Granularity optimization;

2) Multiple materials combination;

3) Fundamental research strengthening for nano-size or chiral absorbing material;

4) Chaff structure improvement and diffusion performance optimization.

\section{REFERENCES}

1. H.J. XIE, J.B. LI, Z.F. XU, T. WANG, Analysis of Wideband Characteristics and Jamming Technology of Chaff Cloud, J. IEEE Trans, 2014: 1053-1057

2. F. JIA, Study on the Preparation and Radar Attenuation Properties of Absorptive Chaff by Wet Chemical Method, Beijing, 2015

3. Y.K. MIAO, B.Y. JIN, The Attenuation of Fiber Cloud to Electromagnetic Wave, in: Electro-Optic Warfare \& Radar Passive Countermeasures, 1997: 31-32

4. B.L. ZHAO, Z. WANG, K.J. RAO, Radar reflection characteristics analysis of multi-orientation iron fiber absorbing materials, in: Chinese Journal of Radio Science, 2004: 280-284

5. S. HU, J.S. LU, T. XU, Effect Evaluation of Chaff Suppress Jamming on Power Attenuation of Radar Signal, in: Journal of Jilin University,2008: 259-262

6. S.W. MARCUS, Electromagnetic Wave Propagation Through Chaff Clouds, J. IEEE Trans,2007: 2032-2042 